\title{
La Teoría de la Complejidad y el Entorno Educativo
}

\author{
María del Consuelo Salgado Soto \\ csalgado@uabc.edu.mx \\ https://orcid.org/0000-0003-2939-9388 \\ Académica de la Facultad de Contaduría y Administración \\ de la Universidad Autónoma de Baja California. \\ Josué Miguel Parra Flores \\ josue.miguel.parra.flores@uabc.edu.mx \\ https://orcid.org/0000-0003-1424-4498 \\ Académico de la Facultad de Contaduría y Administración \\ de la Universidad Autónoma de Baja California.
}

Recepción: 06/09/2021

Aceptación: 22/10/2021

\begin{abstract}
Resumen
La teoría de la complejidad permite explorar a sistemas a partir de su composición como una totalidad. Está ha sido utilizada para modelar organizaciones, fenómenos naturales y sociales, incluso relaciones entre países e individuos; la educación como un fenómeno puede ser considerada un sistema complejo, y ser analizado a través de esta perspectiva con la intención de proponer la aplicación de acciones que modifiquen esa realidad. En este trabajo se analiza un entorno educativo desde el punto de vista sistémico y complejo, para señalar las propiedades o eventos donde se plantea la percepción de un posible conflicto que propicie actuar para resolverlo a partir de la exposición de las interrogantes y acciones pertinentes que definen los factores que modifiquen su realidad a fin de predecir con un grado de confianza las estrategias, políticas o modificaciones en ese entorno.
\end{abstract}

Palabras clave: Complejidad, educación, sistemas complejos y entorno educativo

\section{Abstract}

Complexity theory allows systems to be explored based on their composition. It has been used to model organizations, natural and social phenomena, even relationships between countries and individuals. Education as a phenomenon can be considered a complex system and be analyzed through this perspective with the intention of proposing the application of actions that modify that reality. In this work, an educational environment is analyzed from a systemic and complex point of view to point out properties or events where the perception of a possible conflict that encourages action to resolve it arises from the exposition of pertinent questions and actions that define factors that modify their reality to predict with a degree of confidence strategies, policies, or modifications in that environment. 
Keywords: Complexity, education, complex systems, and educational environment.

\section{Introducción y justificación}

Analizar fenómenos sociales, naturales o biológicos a través de sus componentes básicos o partes fundamentales no permitiría comprender la magnitud o alcance de la realidad donde se presentan. Se requiere de un enfoque holístico, donde se contemplen las propiedades comunes en todos los niveles de una realidad (Von Bertalanffy, 1989) para observar los comportamientos e interacciones de todos sus elementos, es decir, desde el punto de vista sistémico.

Para Lara-Rosano et al., (2017), los sistemas afectan y son afectados por una realidad inmediata a ellos, por consiguiente, una acción que llega del entorno (Medio ambiente) al sistema a través de uno o varios de sus elementos produce una reacción en la totalidad; y a la vez esta reacción provoca alguna modificación en la realidad. Los sistemas existen dentro otros sistemas, donde cada uno de ellos tiene sus características y propiedades. Los cuales se relacionan de una manera no lineal, no determinística y dinámica, con la capacidad de adaptarse a su entorno, además, existe un trabajo colectivo entre sus múltiples componentes, es decir, tienen un comportamiento complejo.

La complejidad, como teoría, estudia situaciones o fenómenos atípicos donde se debe considerar todos los factores interrelacionados en un todo para poder definirlo como sistema y lograr atender conflictos inmersos en ellos.

El presente trabajo se enfoca en una revisión de la teoría de la complejidad para distinguir las características y ventajas de su aplicación, así como una intervención en los sistemas complejos para predecir resultados y en base a ellos definir acciones. La propuesta de aplicación de esta teoría es en el campo de la educación que por sus características y composición puede ser definida como un sistema complejo. Para ello se expone una problemática actual donde se busca atribuir los efectos a ciertos factores del sistema y cómo reacciona el entorno ante ellos.

\section{De la Teoría del Caos a la Complejidad}

\subsection{Teoría del caos}

El caos nos rodea (Lorenz, 1995). Para profundizar en el tema de la teoría de la complejidad, primero se debe comprender la definición básica de caos para diferenciarla de la acepción a la que se enfocara este trabajo, según la Real Academia Española, (2021) es desorden o confusión, en cambio, la concepción de ese término en el área de las ciencias puede ser definido como un comportamiento aparentemente errático o aleatorios de sistemas dinámicos o determinísticas.

La teoría del caos supone una revolución científi$\mathrm{ca}$, se convierte en un área fundamental de las ciencias de la complejidad; esta teoría se enfoca en el proceso de lo que va a suceder en los sistemas dinámicos, en otras palabras, puede considerarse como una filosofía que se encarga de estudiar aquellos sistemas en los que un pequeño cambio puede generar grandes consecuencias, comenta Martínez, (2018).

Por otra parte, para Pidal, (2009) esta teoría trata de atender la relación entre "el orden y el desorden y el orden", esta idea se basa que a partir de un estado de uniformidad ante la presencia de pequeñas perturbaciones se pueden generar acontecimientos futuros impredecibles para llegar en un momento determinado a una estabilidad.

Esta teoría también se relaciona con el efecto mariposa, que al igual describe cómo unos cambios en la condición inicial pueden alterar otra condición en un mediano o largo plazo, por ejemplo, los efectos que pudieran tener el retraso de un vuelo en la cadena de conexiones y cómo los pasajeros se verán afectados, incluso los pasajeros que no están relacionados con dicho vuelo.

Otro ejemplo relacionado es el efecto de un evento de un partido de fútbol en la zona céntrica de una ciudad, en un horario de alto movimiento vehicular, además, que coincide con la hora de salida de la mayoría de los trabajadores en un viernes por la tarde; el impacto de este evento que no se da todos los días, altera el orden del tráfico habitual en ese punto de la ciudad, y de manera gradual va creciendo y extendiéndose hasta llegar a lugares que no están relacionados con el evento deportivo, y en un momento determinado, se presentan acciones que llevan al orden dentro de ese caos, donde los afectados toman decisiones para esquivar las zonas que se encuentra congestionadas o considerar en un futuro posibles acciones para no verse perjudicados por situaciones similares; el efecto mariposa y la teoría del caos ante un pequeño evento causa un resultado caótico, hasta llegar a un estado de equilibrio; como menciona Pidal (2009) del desorden se llega al orden.

La teoría del caos se encarga de estudiar el comportamiento de fenómenos en diversas áreas, entornos o sistemas donde se puede enfocar a lo que posiblemente sucedería, el desorden, esta teoría es una de las bases del surgimiento de la complejidad.

\subsection{Teoría de la complejidad}

Para Morín (2003), la complejidad es el tejido de eventos, acciones, interacciones, retroacciones, 
determinaciones, azares, que constituyen a los fenómenos. El paradigma de la complejidad asegura un marco conceptual que permite establecer relaciones entre diversas disciplinas, promueve y realza un pensamiento metodológico nuevo que aparece como resultado de la organización sistémica, de las interacciones y propiedades no lineales (Barberousse,2008).

La teoría de la complejidad es una forma de demostrar cómo los sistemas se pueden relacionar en términos de la supervivencia, evolución, organización, reorganización, estabilidad y caos. El centro de interés de la complejidad son los objetos sociales, como las organizaciones, o la sociedad misma, definidos por un gran número de elementos interconectados que interaccionan de diversa maneras como afectar, adaptar así como generar nuevos patrones 0 comportamientos.

De acuerdo con Mason, (2008), para comprender la complejidad de una forma más sencilla, esta puede ser revisada con los trabajos relacionados a este paradigma, desde la perspectiva de las grandes mentes como Adam Smith, Friedrich Engels, Alan Turing, Charle Darwin, entre otros, al contribuir a esta teoría a través del estudió de patrones de adaptación y auto-organización, por ejemplo: el comportamiento de las colonias de hormigas y en la construcción de sus nidos, en entender cómo aprende la mente humana, hasta en el interés por comprender el comportamiento de las redes del cerebro; ellos se enfocaron a observar a la totalidad de los elementos de un entorno, que eran relativamente sencillos, en lugar de centrar su investigación a una sola porción del todo, analizaron a esos pequeños sistemas como un todo, considerándolos como adaptativos y complejos, que a su vez mostraban un comportamiento emergente, evolutivo, organizado y diverso.

En su carácter de novedad, la complejidad implica un rompimiento con los supuestos del pensamiento científico moderno; tratada como ciencia introduce un entendimiento en el que se incorporan problemas con características de desorden, a la no-linealidad, temporalidad, emergencia y auto organización, etc., donde estos problemas se encuentran en una realidad difusa en campos de la materia, de la vida o la sociedad (Rodríguez y Leónidas, 2011).

La aplicación de la complejidad, como paradigma científico de investigación, va en aumento en diversas áreas del conocimiento debido a que centra su interés en fenómenos que no son predecibles.

\section{Complejidad y sistemas}

La teoría general de sistemas identifica a los sistemas como un fenómeno que ha invadido todos los campos de la ciencia, incluso, el pensamiento del ser humano. Esta teoría considera que estos sistemas pueden ser socioculturales, biológicos, físicos, tecnológicos, matemáticos, entre otros, además, propone encontrar las propiedades comunes en los sistemas que se presentan en todos los niveles de la realidad, (Von Bertalanffy, 1989). Una de las bases de la complejidad está en el enfoque sistémico.

Todo sistema, sea su origen biológico, físico, social, cultural, etc., no permanecen ni funcionan de manera aislada, sino que pertenecen a una jerarquía conformada por un supra sistema, sistema y subsistemas (Lara-Rosano et al., 2017). Un sistema, explican Ramírez et al., (2019), puede ser identificado como un conjunto de elementos que interactúan entre sí con el fin de lograr un objetivo específico, con influencia mutua para que el comportamiento de cada uno de ellos impacte en otros; asimismo comentan para que un sistema sea concebido como tal, deben identificarse sus partes, las interacciones y las relaciones entre ellas, así como el valor de cada una para sus elementos, también comprender cómo se originan de manera impulsiva para poder simplificar la realidad y llevar a entender los eventos naturales.

Para llegar a una definición exacta de un sistema complejo, lo correcto es preguntarse ¿Qué es? ¿Cuáles son sus elementos básicos? y ¿Cómo está constituido? Las respuestas a estas interrogantes pueden ser una guía para observar la iteración de los sistemas, subsistemas, procesos y demás componentes en el tiempo y espacio del fenómeno, así como la información que se comparte entre ellos. Martínez (2012) comenta que definir un sistema complejo depende de las propiedades del entorno, del fenómeno que se analiza, además, de los comportamientos emergentes que fomentan la retroalimentación, por lo que dar una definición exacta podría considerarse casi imposible puesto que depende del punto de vista de quien establece los factores, criterios y características. Se llega a una definición universalmente aceptada de un sistema complejo a partir de quien lo observa. En un esfuerzo por conceptualizar este tipo de sistema, Maldonado, (2014) comenta que se caracterizan particularmente por una dinámica invariable e impredecible.

Los sistemas complejos están formados por diferentes elementos, también por subsistemas, que interactúan dinámicamente, es decir, que los cambios que se presentan en el tiempo se retroalimentan de manera no lineal, existiendo una relación proporcional entre las causas de unos y los efectos de otros (Ramírez et al., 2019). Son estructuras dinámicas, que evolucionan, se organizan y emergen, compuestas por elementos heterogéneos, donde la función de cada uno tiene repercusión en la vida y comportamiento de todo el sistema. Algunos ejemplos de este tipo de estructura dinámica pueden ser la sociedad, una organización, el gobierno o 
economía de un país, un grupo social o la familia, por mencionar algunos.

Una de las principales características de los sistemas dinámicos y evolutivos es la "Emergencia". Esta característica se basa en la aparición de nuevas propiedades y comportamientos que no están contenidos en un sistema en un inicio. Por lo tanto, no pueden predecirse a partir de los elementos, sino en los cambios que se dan en el tiempo entre las entidades. Estás nuevas características o propiedades se pueden presentar de manera gradual o inmediata.

Una propiedad emergente, define Gershenson (2013) es aquella que se encuentra en un sistema, pero no en sus componentes; esta propiedad se hace presente en las interacciones de los sistemas complejos, no son físicas, pero son reales, en el sentido de que afectan causalmente el futuro.

Con esta descripción podemos concluir que un programa educativo de una universidad es un sistema complejo. Él cual tiene propiedades definidas por el entorno, como perfil de ingreso, competencias, asignaturas, docentes, alumnos, perfil de egreso, pero es necesario que se de la relación entre cada uno de ellos para que ocurra la emergencia como nuevos comportamientos, patrones o propiedades, y así surjan nuevas estructuras en diferentes escalas, por ejemplo, nuevas asignaturas, estancias en organizaciones, contratación de docentes más especializados.

Explica Mason, (2008) que, para lograr el dinamismo, la emergencia, y la evolución, se requiere de un poder inercial o impulso basado en el paradigma de la teoría de la complejidad, que se mantendrá y aumentará su dominio o su espacio en virtud de lo que puede entenderse simple y análogamente como el efecto bola de nieve.

En el contexto de un entorno universitario como un sistema que debe dar respuesta a exigencias y necesidades de la sociedad, los impulsos que recibe este sistema (ver Figura 1), serían los factores que provoquen la evolución, reorganización, formación de nuevas estructuras, patrones y comportamientos como políticas, estrategias para revolucionar e incrementar el progreso de la sociedad.

Figura 1. Universidad y el impulso inercial

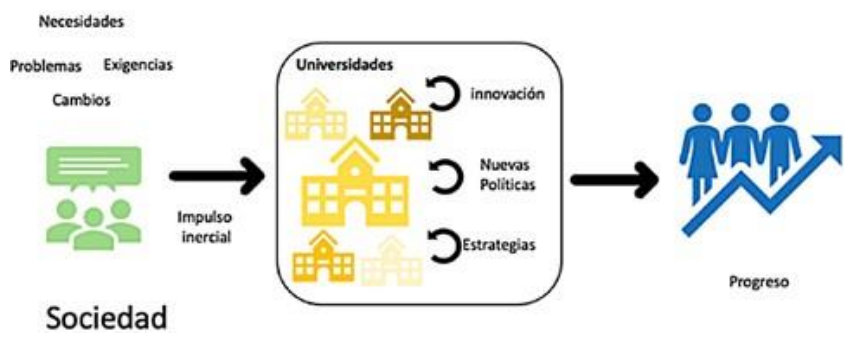

Fuente: Elaboración propia
Ese impulso permitirá que el sistema complejo mantenga $y$, probablemente aumente su propio impulso en él, se genere un caos, en consecuencia, nuevos patrones, relaciones o comportamientos, en un momento determinado de esa dinámica se llegará a la estabilidad, a la definición de alternativas para la toma de decisiones que impactan o se convertirán a la vez en otro impulso inercial para un sistema.

\section{Teoría de la complejidad y cambio en el entorno educativo universitario.}

\subsection{Conceptualización de una institución edu- cativa como un sistema.}

La educación a nivel superior debe atender las demandas de la sociedad mediante una formación exigente y actualizada en el desarrollo de las destrezas en las nuevas generaciones, adicionalmente, estar certificada a través de las instituciones de educación superior que incluyan el conocimiento especializado y la preparación en el área académica como parte del proceso educativo.

Las instituciones de nivel superior son esenciales y cruciales para el desarrollo de las economías. Estas instituciones se interesan en atender temas como la generación del conocimiento, información y la innovación que va en aumento en relación con los problemas sociales, económicos y su crecimiento.

Los problemas sociales, económicos y políticos, son problemas complejos, sin embargo, no pertenecen a la complejidad desorganizada para ser resueltos por métodos definidos que describen comportamientos promedio (Lara-Rosano et al, 2017); para atender y resolver esos problemas implica que se consideren los factores del entorno y comprender las interrelaciones en un todo sistémico, en un contexto de una complejidad organizada.

A partir de la conceptualización y entendimiento de la teoría de la complejidad se analizará al entorno educativo universitario como un ente complejo o una estructura dinámica organizada. Este entorno, definido como un sistema, contiene elementos que incluyen docentes, alumnos, programas educativos, asignaturas, administración, gobierno y sus departamentos o secretarías de educación, estructuras económicas y organizaciones comerciales o sector laboral, entre otros (ver Figura 2).

La intención de observar a este sistema es para proponer acciones que produzcan cambios en el comportamiento y la dinámica mediante una intervención en grados diferentes con impulsos inerciales pero suficientes para que en cada una de estas áreas afecten causalmente su futuro. 
Figura 2. Representación de una institución educativa como un sistema.

Sistema complejo:

institución educativa

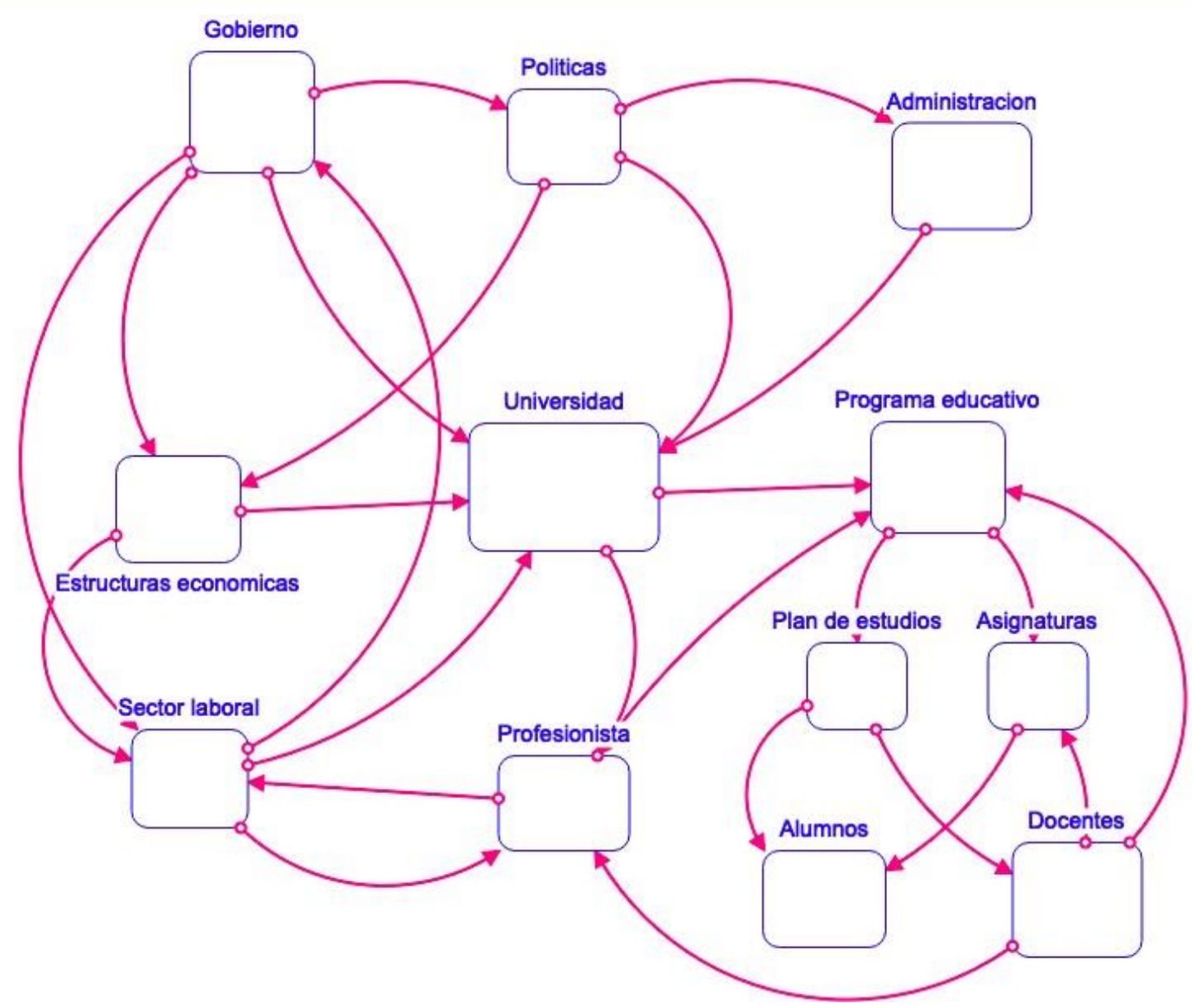

Fuente: Elaboración propia. Software utilizado: Stella Architect.

El cambio en cualquier nivel no es solo consecuencia de efectuar variaciones en un solo elemento en particular sin importar que tan poderosa sea la influencia de ese factor, se trata de generar el impulso total en una nueva dirección prestando atención a tantos factores como sea posible en el sistema en su conjunto.

Antes de tomar una decisión habría que preguntarse ¿Dónde debe generar el impulso? ¿Cuáles son los elementos que tienen influencia en todo el sistema? Se debe profundizar en qué se debe modificar y qué tan grande debe ser el impulso.

Para ello, también se deben considerar factores sociales, económicos, políticos, innovadores o motivacionales para sugerir lo que se puede hacer al respecto como elementos de una estructura, dentro del entorno dinámico.

\section{2 La complejidad y el entorno universitario}

La educación siempre ha sido objeto de muchos estudios realizados desde el punto de vista emocional, conocimiento, laboral, económico, social, tecnológico, etc.

Algunos temas propuestos por diferentes autores están enfocados al análisis del proceso de enseñanza aprendizaje, el impacto de diversos sectores de la sociedad en la educación, o la influencia de las TIC en la enseñanza o en el aprendizaje, y actualmente los estudios que han surgido por la pandemia por COVID-19, por mencionar algunos.

Hay que destacar que se ha utilizado un enfoque que atiende elementos básicos de una situación o fenómeno para comprender o conocer una porción de una realidad, que permite definir estra- 
tegias centradas en componentes simples, específicos, fundamentales o más especializado, que son características del enfoque filosófico Reduccionista (Martínez et al., 2017).

En cambio, desde el enfoque de la teoría de la complejidad, se busca comprender el todo: el proceso de transformación, cooperación, interacción de las funciones y actividades específicas.

En la Figura 3 se muestra la definición de un sistema complejo que incluye elementos como subsistemas y las variables, que recibirán la información del entorno y estas a su vez lo alimentarán. En esta imagen se observa a cada uno de los componentes del sistema escolar universitario, donde cada uno de ellos incluye las posibles variables o factores que recibirá el impulso o energía para lograr el cambio en toda la estructura.

Para lograr un cambio se requiere que en el sistema cambie como una estructura y su naturaleza sistémica del entorno, de tal manera que proporcione resultados significativos y valiosos.

El sistema podría analizarse desde el cumplimiento de los objetivos educativos, la pertinencia de los programas educativos, el aseguramiento del proceso de enseñanza y aprendizaje, las políticas y estructuras educativas, la importancia de un líder escolar, la planta de maestros, el docente comprometido, el estudiante y la adquisición de cono- cimientos y habilidades, los procesos educativos, procesos administrativos universitarios.

En el ámbito externo, pero parte del mismo sistema, también se debe analizar a las estructuras económicas, el entorno familiar del estudiante, organismos de gobierno y comerciales, etc. Son tantos los elementos que puede contener que para saber exactamente qué es lo que está ocasionando una situación en particular, la mejor oportunidad de éxito en el cambio puede radicar en abordar tantos puntos de vista y niveles como sea posible, la respuesta es: abordar el fenómeno desde una perspectiva sistémica, compleja y dinámica.

\subsection{Análisis de un entorno educativo}

Las instituciones de educación superior y sus programas deben responder las necesidades de la sociedad, que incluye al gobierno, entorno laboral y económico (OCDE, 2021).

Los cuestionamientos que hay que tener a consideración son: ¿Qué es lo que requiere la sociedad para seguir progresando? ¿Cuáles son los aspectos del entorno institucional universitario e incentivos necesarios para lograr cumplir con las necesidades que dicta la sociedad? ¿Cómo se puede saber qué hacer con cada uno de estos factores si es imposible y equivocado tratar de aislar y evaluar la importan-

Figura 3. Representación del sistema complejo.

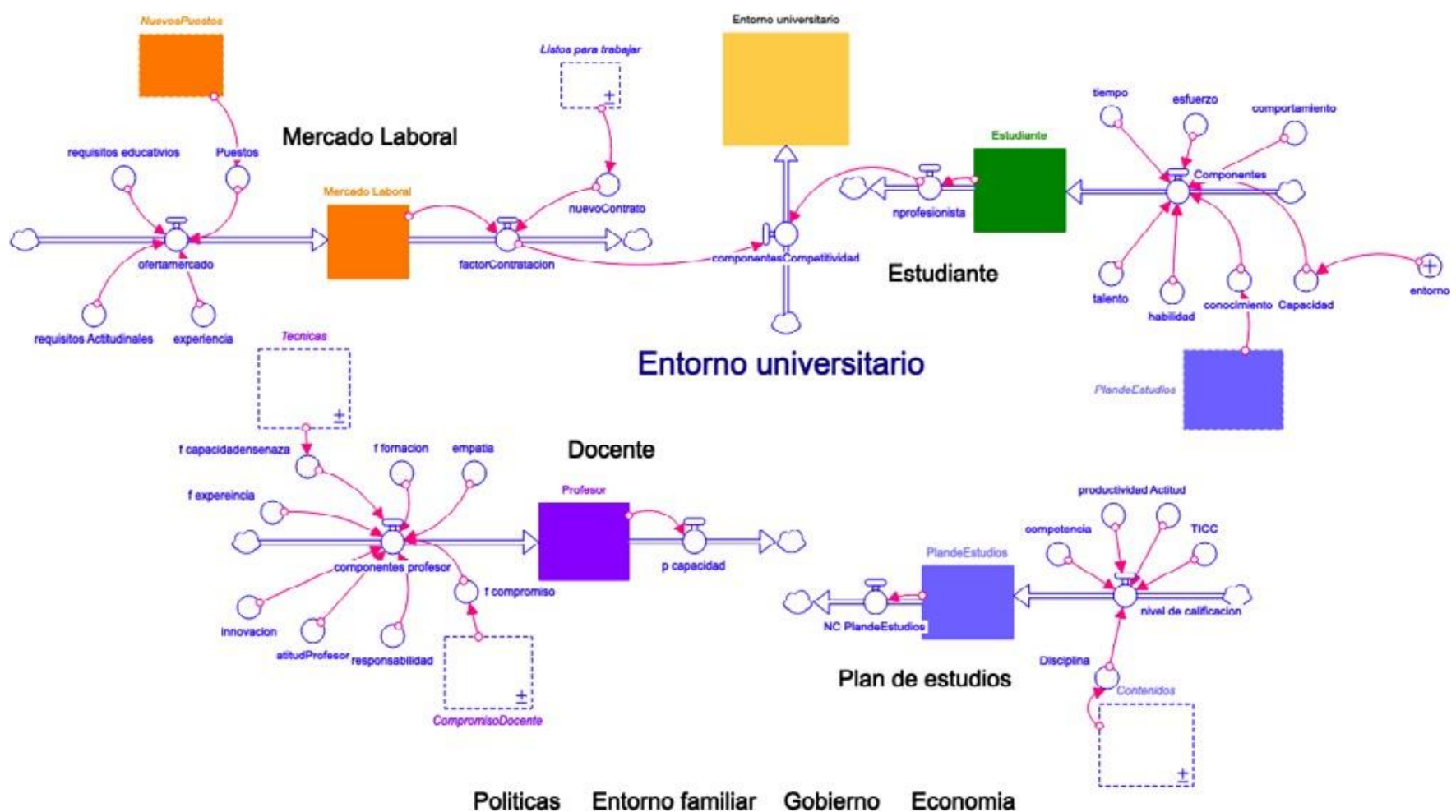

Fuente: Elaboración propia. Software utilizado: Stella Architect. 
Figura 4. Representación y análisis de un entorno educativo.

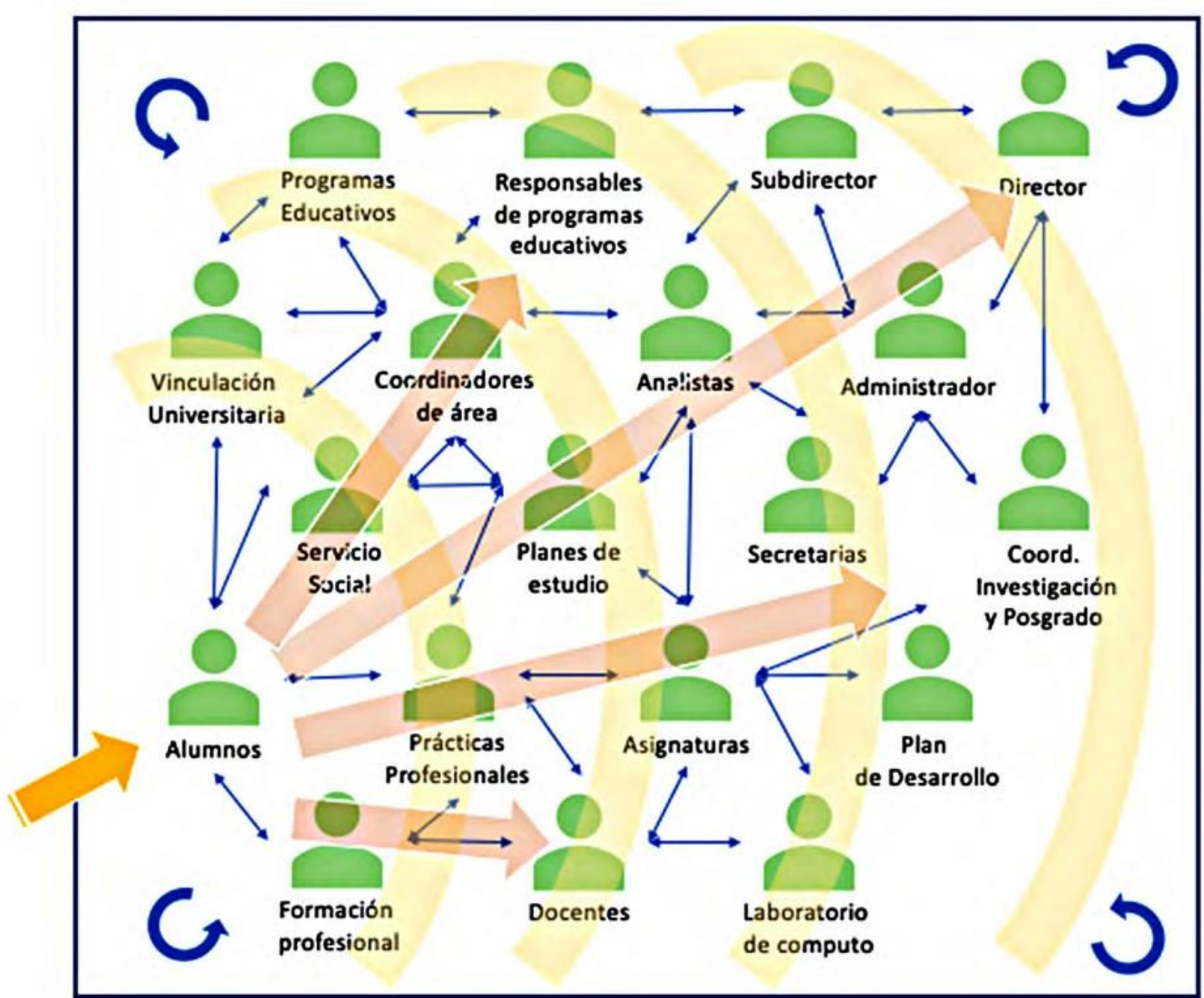

Fuente: Elaboración propia.

cia de cualquier factor? ¿Cómo se puede saber en qué dirección debemos intentar impulsar cualquier factor?

Para analizar un entorno educativo, se parte de un supuesto de que una escuela o facultad de una universidad puede ser concebida como un sistema complejo, heterogéneo, dinámico, a partir de la identificación de sus subsistemas como: la dirección, subdirección, la administración, los programas educativos, responsables de programa, coordinadores de área, los planes educativos, y asignaturas.

Otros subsistemas contemplados son las diferentes coordinaciones como investigación y posgrado, formación profesional, vinculación universitaria, prácticas profesionales, servicio social, el equipo de analistas y secretarias, los responsables del laboratorio de cómputo, entre otras, de igual manera se consideran elementos de este sistema a docentes y alumnos.

Todo como el conjunto de elementos que interactúan con un fin, que no deben ser estudiadas de manera aislada, e identificando la influencia que hay entre ellos en relación al comportamiento y relaciones que se presentan para facilitar el entendimiento de los eventos del sistema.

Después de la revisión realizada al sistema, se plantean las alternativas para decidir cómo generar nueva información. Tal como describe la teoría del caos se debe realizar un pequeño cambio para generar una evolución y emergencia en este sistema; es decir, dar el impulso necesario en ese elemento clave que va a producir los movimientos, que serían los factores que provoquen la evolución, reorganización, patrones y comportamientos como políticas, estrategias, ver la Figura 4.

La imagen anterior aborda un entorno educativo como sistema complejo, el cual contiene los subsistemas y elementos que lo representan. Además, se incluyen las interacciones que se dan entre ellos. Se puede observar que el impulso o energía se dará al elemento clave Alumnos, debido que en la realidad es el factor que provoca los movimientos necesarios en el sistema para generar nuevos acontecimientos. 
El hecho de que los alumnos soliciten un servicio, requieran alguna asesoría, o soliciten algo inesperado al sistema, se provocarán los movimientos necesarios para generar las perturbaciones, que a su vez generan resultados impredecibles así como la posible información que alimente al sistema y al entorno donde está definido. Estos cambios provocados requerirán soluciones emergentes, es decir, soluciones no estructuradas dando pie a la aparición de nuevas estructuras o propiedades.

\section{Conclusiones}

La educación es un sistema complejo dado a que intervienen muchos aspectos para tratar de comprenderla. Una manera de analizar el fenómeno de educación o entornos educativos es utilizando una metodología inspirada en la teoría de la complejidad. Ya que al analizar sólo sus componentes de manera aislada se perderían las relaciones existentes entre el medio ambiente y los elementos que lo componen, provocando que no podamos observar una fotografía completa del problema estudiado.

La complejidad en el entorno educativo permite identificar a los agentes que intervienen y cómo se relacionan entre ellos, con el objetivo de generar y observar cambios que se dan de manera exponencial, no-lineal, dinámica y emergente. Así como la integración de nuevos subsistemas, grupos o estructuras a fin de permitir una adaptación a los cambios del medio ambiente para lograr una estabilidad y un equilibrio.

La teoría de la complejidad aplicada en el ámbito educativo puede ser usada como una metodología para crear herramientas tecnológicas, software o simulaciones capaces de ayudar al entendimiento de los patrones de comportamiento y las interacciones presentes en el ámbito educativo.

Actualmente, se necesitan nuevas herramientas capaces de reflejar los cambios en el medio ambiente de un ámbito educativo que ayuden a predecir los nuevos comportamientos generados y propiedades emergentes que puedan surgir; esto podría propiciar la participación de los agentes involucrados para el diseño de nuevas políticas educativas o administrativas. Además de estrategias curriculares o la mejora del aprendizaje de manera efectiva en donde actualmente no lo es.

Mediante una intervención masiva y sostenida en todos los niveles del sistema, se podrán conocer las proyecciones como consecuencia de los movimientos para predecir con un grado de confianza la dirección de mejora de los temas relacionados con ese ecosistema. La teoría de la complejidad evita las certezas y promueve la incertidumbre para impulsar un cambio.

\section{Referencias}

Barberousse, P. (2008). FUNDAMENTOS TEÓRICOS DEL PENSAMIENTO COMPLEJO DE EDGAR MORIN. Revista Electrónica Educare, XII(2),95-113. ISSN:14094258. Disponible en: https://www.redalyc.org/articulo.oa?id=194114586009

Gershenson, C. (2013). ¿Cómo hablar de complejidad?. Llengua, Societat I Comunicació, 11. https://doi.org/https://doi. org/10.1344/LSC-2013.11.3

Lara-Rosano, F., Gallardo, A., \& Almanza, S. (2017). Teorías, métodos y modelos para la complejidad social. Ciudad de México: COLOFÓN Ediciones Académicas.

Lorenz, E. (1995). The Essence of Chaos. University of Washington Press.

Maldonado, C. (2014) ¿Qué es un Sistema Complejo? Revista Colombiana de Filosofía de la Ciencia. 14 (29): 71-93, https:// www. redalyc. org/articulo. oa?id $=41438646004$.

Martínez, C. (2018). Teoría del Caos y Estrategia Empresarial. Tendencias, 19(1). 204-214

Martínez, G. (2012). Sistemas complejos, Revista.unam.mx, Vol 13 (4). Disponible en: http://www.revista.unam.mx/vol.13/ num4/art44/art44.pdf.

Martínez M., García, E. Bernal, C. (2017) Reduccionismo, leyes naturales y complejidad diferentes estrategias de investigación y explicación científica. Scientiae Studia, Sao Paulo, V 15, núm 2, pag. 243-262.

Mason, M. (2008). What Is Complexity Theory and What Are Its Implications for Educational Change?. In M. Mason, Complexity Theory and the Philosophy of Education. Wiley-Blackwell.

Morin, E. (2003). Introducción al pensamiento complejo, BarceIona, Gedisa.

OECD. (2021). Los países deben hacer que la profesión docente sea más atractiva desde el punto de vista financiero e intelectual. Recuperado de https://www.oecd.org/centrodemexico/ medios/lospaisesdebenhacerquelaprofesiondocenteseamasatractivadesdeelpuntodevistafinancieroeintelectual.htm.

Pidal, M. (2009). La teoría del Caos en las Organizaciones. Cuadernos Unimetanos.

Ramirez, M., Lara-Rosano, F., Rosales, R., Manrique, E., Ramirez, H., \& Maldonado, G. (2019). Multi-Agent Complex System of Identification of Characteristics and Personality Types and Their Relationships in the Process of Motivation of Students. In G. Jezic, Y. Chen-Burger, M. Kus ek, R. S perka, R. Howlett \& L. Jain, Agent and Multi-agent Systems: Technologies and Applications 2019 (13th ed., pp. 143-152). Springer. Retrieved 2 November 2021, from.

Real Academia Española. (2021). Caos. REAL ACADEMIA ESPAÑOLA: Diccionario de la lengua española (23rd ed.).

Rodríguez, L., y Leónidas, J. (2011). TEORÍAS DE LA COMPLEJIDAD Y CIENCIAS SOCIALES Nuevas Estrategias Epistemológicas y Metodológicas. Nómadas. Revista Crítica De Ciencias Sociales Y Jurídicas, 30(2).

Von Bertalanffy, L. (1989). Teoría General de los Sistemas. México: Fondo de Cultura Económica. 